\title{
Monte Carlo simulation on breast cancer detection using wire mesh collimator gamma camera
}

\begin{abstract}
This paper presents the preliminary results of the new low energy high resolution wire-mesh collimator gamma camera in mapping breast cancer cells, by employing $140 \mathrm{keV}$ photons of Technetium-99 m radionuclide tracer. The complete model of photons propagation and detection, as well as the human cells activities are simulated using Monte Carlo N-Particle code. Abnormal cells of different tumor to background values are investigated, and the results from the conventional collimator and wire-mesh collimator are compared. The results are evaluated in terms of the collimator sensitivity and the contrast to background ratio. In our assessment, the wire mesh collimator gamma camera yields slightly better results than the multihole collimator for sensitivity, however produces insignificant performance in the contrast to background evaluation.
\end{abstract}

Keyword: Breast cancer; Monte Carlo; Wire mesh collimator 